\title{
Molecular evidence for the nuclear localization of FADD
}

\author{
M Gómez-Angelats ${ }^{1,2}$ and JA Cidlowski ${ }^{\star, 1}$ \\ 1 The Laboratory of Signal Transduction, Molecular Endocrinology Group, \\ National Institute of Environmental Health Sciences, National Institutes of \\ Health, 111 Alexander Drive, Research Triangle Park, NC 27709, USA \\ 2 Present address: Almirall Research Centre, Cardener 68-74, 08024 \\ Barcelona, Spain; e-mail: mgomeza@almirallprodesfarma.com. \\ * Corresponding author: JA Cidlowski. Tel.: 919-541-1564; Fax: 919-541-1367; \\ E-mail: cidlowski@niehs.nih.gov
}

Received 12.11.02; revised 10.1.03; accepted 12.2.03 Edited by G Melino

\begin{abstract}
The Fas-associated death domain (FADD) adaptor protein FADD/Mort-1 is recruited by several members of the tumor necrosis factor receptor (TNFR) superfamily during cell death activated via death receptors. Since most studies have focused on the interaction of FADD with plasma membrane proteins, FADD's subcellular location is thought to be confined to the cytoplasm. In this report, we show for the first time that FADD is present in both the cytoplasm and the nucleus of cells, and that its nuclear localization relies on strong nuclear localization and nuclear export signals (NLS and NES, respectively) that reside in the death-effector domain (DED) of the protein. Specifically, we found that a conserved basic KRK ${ }^{35}$ sequence of the human protein is necessary for FADD's nuclear localization, since disruption of this motif leads to the confinement of FADD in the cytoplasm. Furthermore, we show that the leucine-rich motif LTELKFLCL ${ }^{28}$ in the DED is necessary for FADD's nuclear export. Functionally, mutation of the NES of FADD and its seclusion in the nucleus reduces the cell death-inducing efficacy of FADD reconstituted in FADD-deficient T cells.
\end{abstract}

Cell Death and Differentiation (2003) 10, 791-797. doi:10.1038/ sj.cdd. 4401237

Keywords: FADD/Mort-1; FAS; nuclear localization signal; nuclear export signal

Abbreviations: TNFR, tumor necrosis factor receptor; NLS, nuclear localization signal; NES, nuclear export signal; DED, death-effector domain; DD, death domain.

\section{Introduction}

Programmed cell death induced by the interaction of cell surface death receptors with their respective extracellular ligands plays a central role in numerous life and death decisions ranging from embryonic development to cellular homeostasis. In mammals, the intracellular signaling initiated by death receptors of the tumor necrosis factor receptor (TNFR) superfamily leads to the activation of hierarchically organized intracellular signaling pathways that typically culminate in the death of cells bearing such receptors. ${ }^{1}$ Fas-associated death domain (FADD) is an adaptor death domain (DD)-containing protein of approximately $25 \mathrm{kDa}^{2}$ shared by several death receptors that couple death signals to the intracellular death machinery. The human protein consists of 208 amino acids arranged in 12 amphipathic helices functionally divided into six helices in the death-effector domain (DED), which is located at its $\mathrm{NH}_{2}$-terminus, and six helices that form the $\mathrm{DD}$ at the $\mathrm{COOH}$-terminus. ${ }^{3}$ Mechanistically, DD-containing adaptor proteins like FADD or TNFR1-associated DD (TRADD) appear to be required for cell death signaling induced by DD-containing receptors such as Fas/CD95, TNFR1, and TRAIL 1 and TRAIL 2 receptors. ${ }^{2,4,5}$ The best characterized signaling mechanism mediated by FADD is the formation of the death-inducing signal complex (DISC) in which, upon activation of death receptors, FADD interacts with the intracellular DD of the receptors and/or other adaptor proteins such as TRADD, ,2,6,7 and the DED of FADD subsequently recruits and activates caspase-8. ${ }^{8}$ The intervention of FADD in this mechanism is primordial to signal death because expression of a dominant-negative form of FADD consisting of the DD alone (DN-FADD) impairs Fas and TNFR1 death signaling cascades. ${ }^{9}$ Moreover, FADD ${ }^{-/-}$knockout mice display profound defects in apoptotic pathways, particularly in the immune system. ${ }^{10}$ In addition to its prominent role in cell death, FADD may have a role in cell-cycle control and proliferation of lymphoid cells, ${ }^{10-13}$ as well as embryonic development. ${ }^{14}$ FADD's mode of action is assumed to take place in the cytoplasm, possibly because the majority of studies document the interaction of FADD with plasma membrane receptors and associated proteins. However, the cellular biology and regulation of FADD, its dynamics, as well as the understanding of cellular processes governed by FADD, remain poorly understood. One level of the regulation of FADD in mammalian cells was shown to occur via its phosphorylation at the serine-194 by a nonidentified $70-\mathrm{kDa}$ cell-cycle-related kinase, ${ }^{15}$ but the biological significance of FADD phosphorylation is still a conundrum since both forms of FADD can interact with activated death receptors.

In this report, we provide new experimental evidence for the presence of FADD in the nucleus and the existence of functional nuclear localization signals (NLSs) in the aminoacid sequence of FADD. We propose that the mechanism of action of FADD needs to be reconsidered as a result of its nucleocytosolic localization. This concept will undoubtedly help to resolve questions on functions regulated by this signaling adaptor molecule. 


\section{Results and discussion}

We have detected surprising constitutive expression of FADD in both the cytoplasm and nuclear compartments in a spectrum of cell lines examined by confocal microscopy. HeLa, HEK 293, Jurkat, HTC, COS-1, and A549 cells were immunostained with a monoclonal lgG1 directed against the DD of FADD or with a polyclonal antibody that recognizes the phosphorylated form of FADD (data not shown, and Figure 1A). These cell lines examined exhibited FADD concentrated in the nucleus of the cells (Figure $1 \mathrm{~A}$, panels $a, b, d, f)$. In contrast, we observed that in Jurkat T-lymphocytes approximately $50 \%$ of the cell population harbored FADD in the nucleus, whereas the other $50 \%$ displayed FADD in the cytoplasm (Figure 1Ac). The use of this monoclonal anti-FADD has been validated and well documented in specific studies for its ability to recognize (1) native and denatured FADD, (2) FADD recruited in the DISC of Fasactivated cells, and (3) the dominant-negative form of FADD termed DN-FADD. ${ }^{16-19}$ The presence of FADD in the nucleus was confirmed by the examination of the expression of FADD in protein samples from nuclear and cytosolic fractions extracted from HeLa cells (Figure 1B) in which FADD exhibited a nuclear/cytosolic pattern of expression similar to that found for transcriptional activators and other nuclear proteins. $^{20}$
A

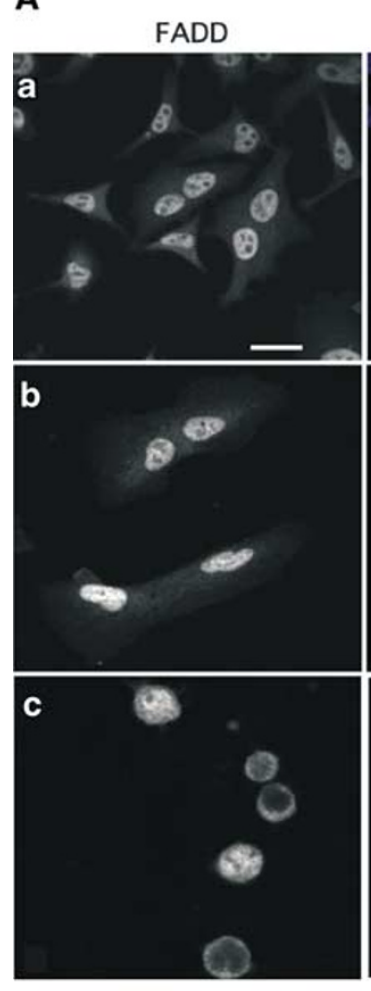

Hoescht

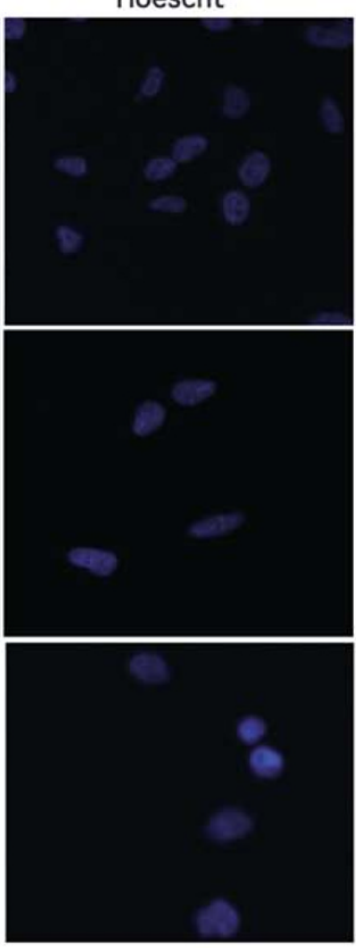

FADD
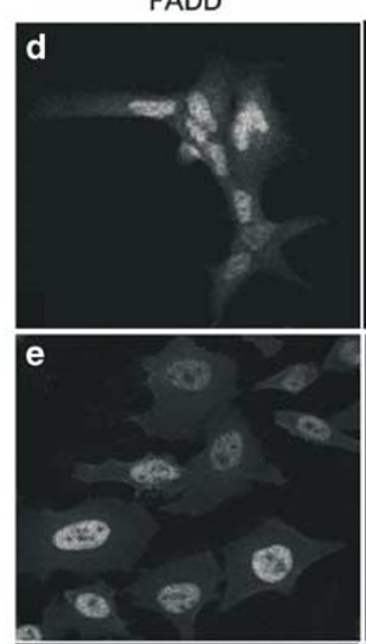

f

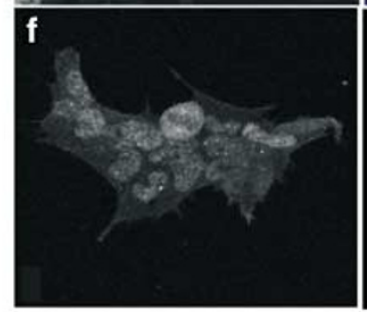

Hoescht
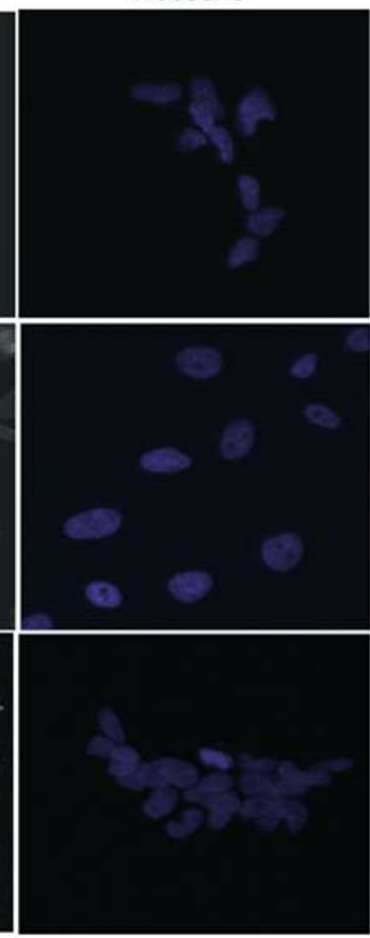

B

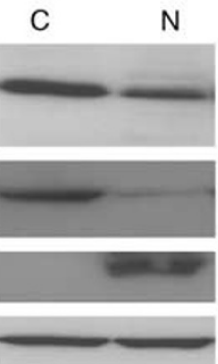

FADD

\section{$\beta$ TUBULIN}

LAMIN B

ACTIN

Figure 1 FADD is endogenously expressed in the cytosol and the nucleus. (A) Immunofluorescence imaging of cellular distribution of endogenous FADD examined by confocal microscopy in HeLa (a and e), A549 (b), Jurkat (c), and HEK 293 ( $d$ and f) cells for FADD shown in left panels, and their respective nuclear (Hoechst) staining shown in right panels. Cells were fixed and exposed to monoclonal anti-FADD IgG plus Alexa Fluor 594-tagged anti-mouse (a-d) and rabbit polyclonal anti-FADD plus FITC-tagged anti-rabbit (e and f). (B) Detection of FADD expression in cytosolic and nuclear fractions extracted from HeLa cells. Each lane was loaded with equal amount of total protein $(20 \mu \mathrm{g})$ and subjected to Western blot using monoclonal anti-FADD. Immunoblots were stripped and reprobed for B-tubulin and lamin B as cytosolic and nuclear markers respectively, and for actin as a marker of total amount of protein per lane 
Since its discovery, FADD has been reported to exert its function primarily by signaling at the plasma membrane; ${ }^{2,21}$ therefore, our finding that FADD displays nuclear localization was unprecedented. Examination of the primary amino-acid sequence of full-length FADD (Figure 1C) led us to the identification of one putative NLS located in Helix 3 of the DED. The NLS of FADD corresponded to a highly conserved short stretch of three basic amino acids $\mathrm{KRK}^{35}$ (Figure 2) that resembled a functional monopartite NLS of other wellcharacterized nuclear proteins such as Myc. ${ }^{22}$

To define whether the $\mathrm{KRK}^{35}$ motif of FADD was a functional NLS, a mutant form of FADD (NLS Mutant-FADD) was generated by replacing the $\mathrm{KRK}^{35}$ with the sequence $A A A^{35}$ through site-directed mutagenesis. HeLa cells were transfected with either wild-type (WT)-FADD or NLS MutantFADD, and were immunostained with FADD- and Alexa Fluor594-conjugated antibodies. Overexpression of fulllength FADD in HeLa cells resulted in the appearance of spread filament-like structures as observed by confocal microscopy (Figure 3A), similar to those reported by Siegel et al. ${ }^{23}$ that were attributed to the formation of death-effector filaments characterizing the overexpression of most DEDcontaining proteins. In most cells overexpressing FADD, the fluorescence was no longer concentrated in the nucleus (Figure 3Aa) compared to our observations for the endogenous FADD (Figure 1A), perhaps suggesting that the formation of filamentous structures may be an artefact of overexpression. Nonetheless, expression of the NLS Mutant-FADD in the cells led to a strong exclusion from the nucleus (Figure $3 A b$ ) and formation of less apparent, filament-like structures. These data suggest that the basic $\mathrm{KRK}^{35}$ sequence in the DED of FADD is required for FADD's subcellular distribution and its nuclear localization.

To determine whether the presence of FADD in the nucleus is dependent on nucleocytosolic trafficking, we scrutinized the primary sequence of FADD for putative leucine-rich motifs with conserved spacing and hydrophobicity that are characteristic of nuclear proteins. Analysis of the primary aminoacid sequence of FADD revealed the existence of one consensus $\left[\mathrm{LX}^{2-3} \mathrm{LX} \mathrm{X}^{2-3} \mathrm{LXL}\right.$; see Nakielny and Dreyfuss ${ }^{24}$ for review) leucine-rich nuclear export signal (NES) located in the DED of human FADD formed by the sequence LTELKFLCL $^{28}$ (Figure 2), which conforms to a rev-type consensus NES as that found in HIV-1 Rev. ${ }^{25}$ Using sitedirected mutagenesis we eliminated the leucine-rich motif of FADD, and generated an NES Mutant-FADD mutant harboring the amino-acid sequence ATEAKFACA ${ }^{28}$. Consistent with

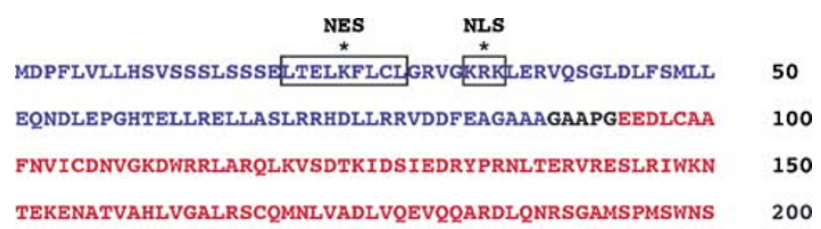

DASTSEAS

Figure 2 Primary structure of human FADD with the identified putative nuclear localization and nuclear export motifs (NLS and NES, respectively). Amino acid sequence in blue and red color represent the DED and DD of FADD, respectively the hypothesis that FADD is transported to the nucleus, HeLa cells transfected with the NES Mutant-FADD showed significant retention of FADD in the nucleus despite overexpressing this protein (Figure $3 \mathrm{Ac}$ ). This observation was in marked contrast to the pattern observed with overexpressed WT-FADD or the NLS Mutant FADD (Figures $3 A a$ and $3 A b$, respectively), supporting the premise that FADD is shuttled into the nucleus, and that the NES sequence in FADD is functional and necessary for FADD's nuclear export.

To validate and extend these results, green-fluorescent protein (GFP)- tagged FADD was generated by linking GFP to the $\mathrm{N}$-terminus of FADD to assess the subcellular distribution of GFP fusion proteins in living cells by localizing direct fluorescence from the GFP moiety. Expression of GFP-FADD in HeLa cells led to a heterogeneous pattern of cytosolic and nuclear fluorescence among cells (Figure $3 \mathrm{Bb}$ ), while both HEK 293 and A549 cells displayed a significant amount of green fluorescence in the cytoplasm (Figure $3 \mathrm{Cb}$ and $3 \mathrm{Cf}$, respectively). It should be noted that the fusion of GFP with FADD yields a protein of approximately $52 \mathrm{kDa}$, considerably higher than the molecular size of endogenous FADD present in the cells, which may compromise trafficking mechanisms of this overexpressed fusion protein. However, in every cell type examined for the expression of GFP-NLS Mutant-FADD, all the green-fluorescent cells exhibited profound cytoplasmic staining and a loss of nuclear fluorescence (Figures $3 \mathrm{Bc}, \mathrm{Cc}$ and $\mathrm{Cg}$ ) compared with their respective controls, supporting the notion that a $\mathrm{KRK}^{35}$ cluster defines a functional NLS necessary for the normal shuttling of FADD to the nucleus. In contrast, confocal analyses of cells transfected with GFPNES Mutant-FADD (Figures 3Bd, Cd and Ch) showed a prominent increase in the intensity of green fluorescence in the nucleus. Thus, GFP-NES Mutant-FADD is actively transported into the nucleus in the absence of this unique leucine-rich sequence.

Overexpression of FADD in cells has been reported to induce apoptosis owing to FADD oligomerization and the association of FADD molecules with plasma membrane death receptors in the absence of death receptor ligand. ${ }^{26}$ Although overexpression of FADD in HeLa, HEK 293, and A549 cells induced cell death, the percentage of death detected at the time of data acquisition ( $24 \mathrm{~h}$ post-transfection) was negligible, in contrast to the $80 \%$ cell death found in Jurkat T- cells after $24 \mathrm{~h}$ of cell transfection with FADD (data not shown). In lymphoid cells, the interaction of FADD with death receptors constitutes an important step of the immune response. ${ }^{27}$ Since induction of lymphocyte apoptosis through cell death receptors is a physiologically well-characterized model of apoptosis, we examined whether the mutational changes that we introduced in FADD exerted any influence in FADD's ability to trigger cell death. For these experiments, we used the FADD-deficient Jurkat T-cell line ${ }^{28}$ which is resistant to apoptosis induced by the Fas/CD95 receptor. Since oligomerization of FADD following overexpression has been reported to induce both apoptosis and necrosis, ${ }^{26}$ we quantified apoptotic cell death by measuring cell shrinkage, a distinctive feature of apoptotic cell death. ${ }^{29}$ Cell shrinkage was examined by fluorescence-activated cell sorting (FACS) analyses of the forward-scatter light in the gated greenfluorescent cells expressing the GFP-FADD proteins that 

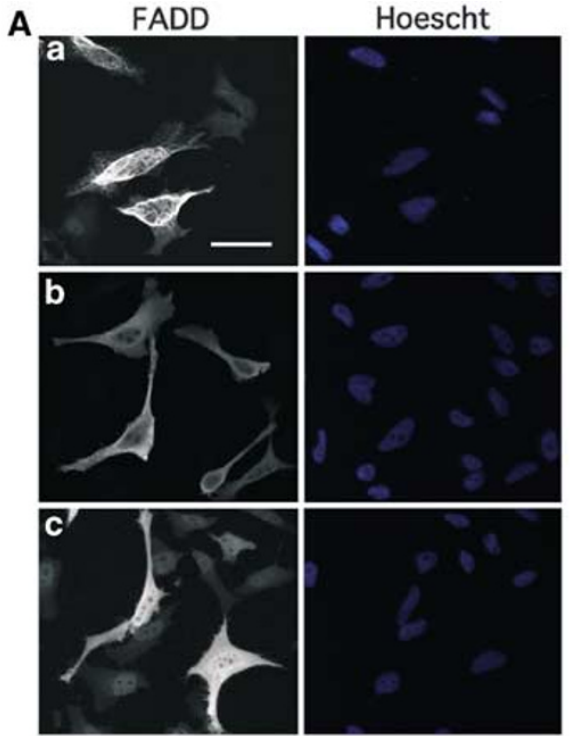

B
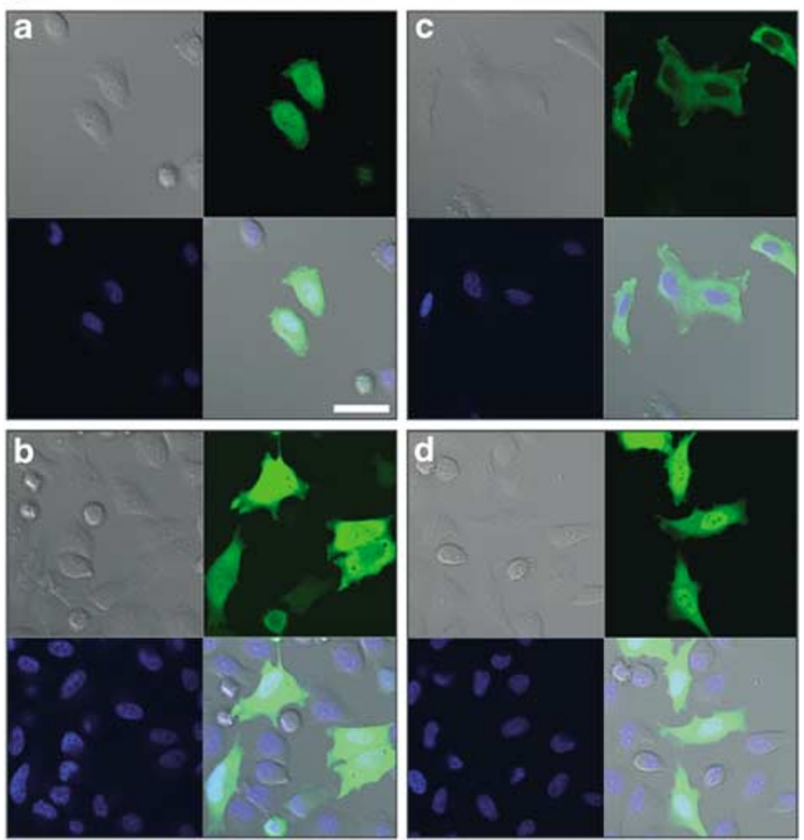

disposable $0.4 \mathrm{~cm}$ gap BTX cuvettes (Genotronics, Inc.), and mixed with $10 \mu \mathrm{g}$ of each of the plasmids. Electroporation was performed by setting the BTX ECM 600 electroporator at $260 \mathrm{~V}, 1040 \mu \mathrm{F}$, and $720 \Omega$. After transfection, cells were immediately placed in six-well plates containing $6 \mathrm{ml}$ of complete medium per well, and were used $18 \mathrm{~h}$ after transfection. Transfection efficiency was routinely measured by FACS and was determined to be between 25 and $40 \%$. For apoptosis analyses, the green-fluorescent populations of cells were gated for each of the groups. Adherent cell cultures were plated $24 \mathrm{~h}$ prior to transfection and were allowed to grow to $50-70 \%$ confluence. Cells were washed with Opti-MEM (Life Technologies, Inc.) and transfected using Fugene-6 (Roche, Germany) according to the manufacturer's recommendations. 

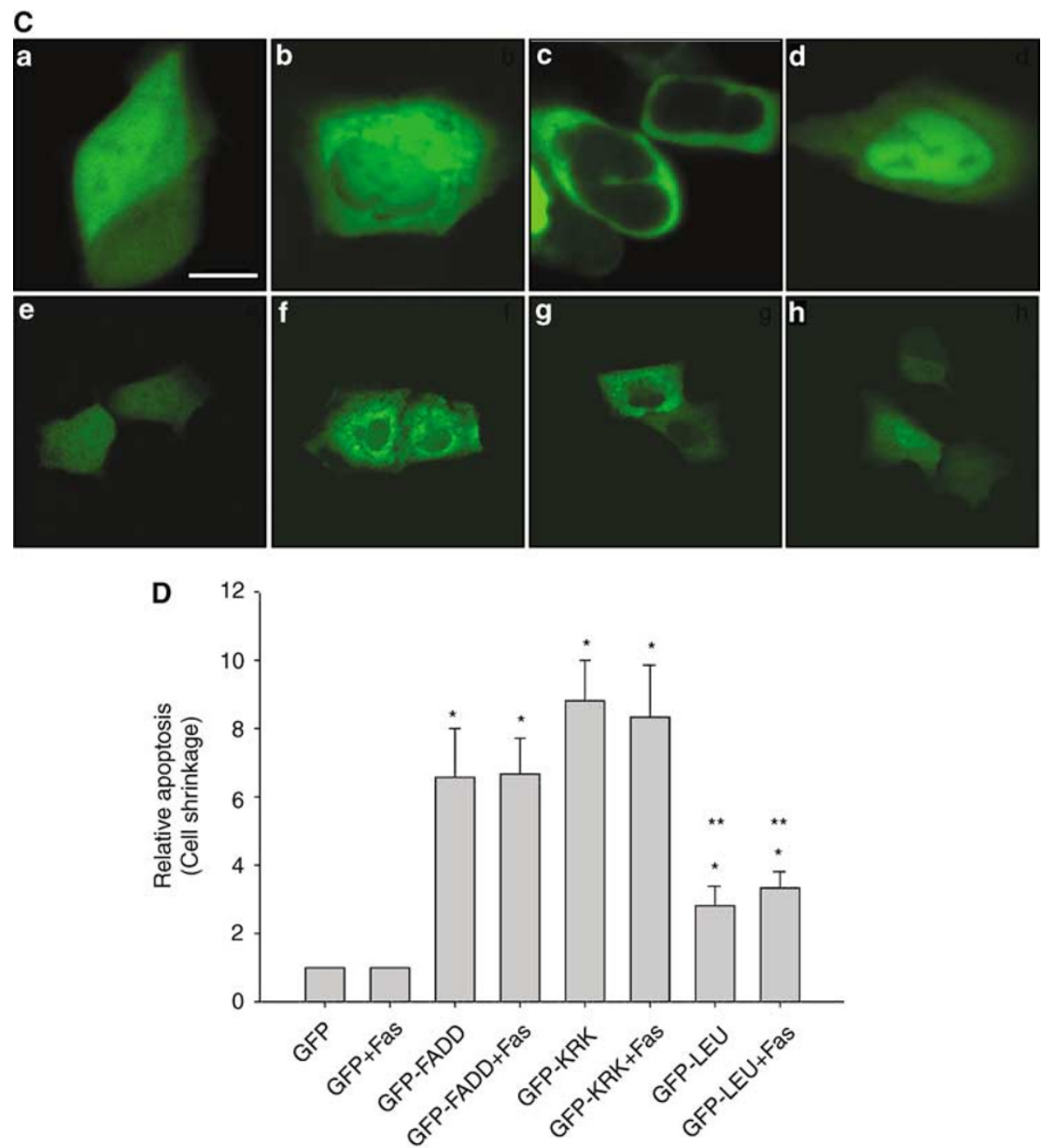

Figure 3 Effect of mutational elimination of the NLS and NES of FADD. (A) Images show the immunofluorescence staining of HeLa cells with anti-FADD IgG after $24 \mathrm{~h}$ of transfection using, WT-FADD (a), NLS Mutant-FADD (b), NES Mutant FADD (c) constructs examined by confocal microscopy. Right panels show confocal images of Hoechst staining for each sample. (B) Subcellular distribution of GFP-FADD in living HeLa cells. Confocal images of cells expressing pEGFP-C2 empty vector (a), GFPFADD (b), GFP-NLS Mutant-FADD (c), and GFP-NES Mutant-FADD (d) mutant with transmitted light image (top left), green fluorescence (top right), Hoechst (bottom left) and superimposition of the three above per each of the preparations. (C) Subcellular distribution of GFP-FADD in living HEK 293 and A549 cells transfected with pEGFP-C2 (a and e, respectively), GFP-FADD ( $b$ and f, respectively), NLS Mutant-FADD (c and g, respectively), NES Mutant-FADD (d and h, respectively). Apoptosis induced by reconstitution of FADD in human FADD-deficient T cells. A total of 10 million FADD-deficient cells were transfected with each of the $p E G F P$-C2, GFP-FADD, GFP-NLS Mutant-FADD or GFP-NES Mutant-FADD constructs. At $18 \mathrm{~h}$ after transfection, cells were resuspended in fresh medium and were exposed to $500 \mathrm{ng} / \mathrm{ml}$ of anti-Fas IgM CH-11 for $1 \mathrm{~h}$. Cells were harvested and subjected to FACS analysis by gating the green-fluorescent cell population and analyzing the percentage of green cells displaying a decrease in cell volume. For each treatment, data was expressed as fold increase of apoptosis over that detected in the GFP-FADD-transfected cells in each experiment. Results are presented as the mean \pm S.E. of three different experiments. . Significance with $P<0.01$ versus GFP group; ${ }^{* *}$ Significance with $P<0.05$ versus GFP-FADD group

The precipitates were incubated with cells in Opti-MEM medium, which was replaced by complete medium after $4 \mathrm{~h}$ of transfection. Cells were maintained at $37^{\circ} \mathrm{C}$ and $5 \% \mathrm{CO}_{2}$, 95\% air until analyses.

\section{Preparation of DNA constructs and site-directed mutagenesis}

pcDNA3 vector containing the full length of FADD was kindly provided by Dr. A Strasser (Melbourne, Australia). To generate GFP-tagged FADD, we excised the cDNA of FADD from the pcDNA3-FADD vector through digestion with $\mathrm{Bam} \mathrm{H} 1$ and Kpn1 and subcloned into the multiple cloning site of BamH1/Kpn1-digested pEGFP-C2 (BD Biosciences Clontech, Palo Alto, CA, USA). Plasmid DNA was prepared by using Qiagen Maxiprep kits (Valencia, CA, USA).

Site-specific mutagenesis was introduced by PCR with the QuikChange kit (Stratagene, La Jolla, CA, USA) by following the manufacturer's instructions and using pcDNA3-FADD or GFP-FADD as templates. Oligonucleotides were individually designed following the QuikChange manufacturer's recom- 
mendations and were synthesized by Integrated DNA Tech. (Coralville, IA, USA). Mutations were verified by PCR with the BigDye $^{\mathrm{TM}}$ Terminator Cycle Sequencing kit (Applied Biosystems, Foster City, CA, USA) and analysis were performed at the NIEHS Sequencing Core (Research Triangle Park, NC, USA). For elimination of the putative NLS, $\mathrm{KRK}^{35}$ was mutated to $A A A^{35}$ by using the oligonucleotide sequence $5^{\prime}$ GC CTC GGG CGC GTG GGC GCG GCC GCG CTG GAG CGC GTG CAG AG $3^{\prime}$ and its reverse complementary. This mutant is designated NLS Mutant FADD. For destruction of the sequence LTELKFLCL, leucines were mutagenized to alanines by using the primer $5^{\prime} \mathrm{CG}$ AGC AGC GAG GCG ACC GAG GCC AAG TTC GCA TGC GCC GGG CGC GTG GG3' and its reverse complementary. This mutant is designated NES Mutant FADD.

\section{Cell fractionation and Western blot analyses}

Cytoplasmic and nuclear extracts were prepared by standard methods with a fractionation kit (Biovision, CA, USA), and $20 \mu \mathrm{g}$ of each denatured protein sample was analyzed by Western blot as previously described. ${ }^{17}$ Antibodies for tubulin (Upstate Biotechnology, NY, USA) and lamin B (Zymed, CA, USA) were used as positive controls for the cytosolic and nuclear fractions, respectively. Results were confirmed with commercially available fractionated nuclear extracts $(4 \mathrm{C}$ Biotech, Belgium; not shown).

\section{Immunostaining and confocal microscopy}

HeLa, HEK 293, and A549 cells were plated and transiently transfected on glass-bottom culture dishes (MatTek Corporation, Ashland, MA, USA) for live imaging, and on two-well LabTek chamber slides (Nalge Nunc International, Naperville, IL, USA) for immunostaining. To prepare cells for immunofluorescence, monolayers of HeLa, HEK 293, A549, and pelleted Jurkat cells were rinsed in phosphate-buffered saline (PBS) and fixed for $10 \mathrm{~min}$ at room temperature in freshly prepared $4 \%(w / v)$ paraformaldehyde (Sigma, St. Louis, MO, USA). Cells were then permeabilized with $0.2 \%$ Triton $X-100$ and treated with $4 \%$ BSA-PBS solution as a blocking reagent. Cells were exposed to mouse anti-FADD (BD BiosciencesPharmingen, CA, USA), or rabbit antiphospho-ser194-FADD (Cell Signaling Technologies, Beverly, MA, USA) diluted 1:50 and $1: 300$, respectively, in a $1 \%$ BSA-PBS solution and were incubated overnight at $4^{\circ} \mathrm{C}$. After three washes with PBS, Fluor594 ${ }^{\circledR}$-conjugated anti-mouse or FITC-conjugated antirabbit (Molecular Probes, Eugene, OR, USA) were diluted in $1 \%$ BSA-PBS and added to the cells for $1 \mathrm{~h}$. After three washes with PBS, the upper chamber portions of the slides were removed, and the fixed cells were mounted and preserved with Prolong Anti-Fade (Molecular Probes). Negative controls for staining consisting of fixed cells exposed to either Alexa-Fluor594 ${ }^{\mathbb{R}}$-conjugated IgG or FITC-conjugated were done to confirm specificity. For live GFP-tagged imaging, cells were analyzed $18 \mathrm{~h}$ post-transfection, and the DNAspecific fluorochrome Hoechst 33342 (Molecular Probes) was occasionally added to the cells to visualize nuclei.

Images from immunostaining and live imaging were collected by using a Zeiss inverted laser scanning confocal microscope LSM 410 UV (Zeiss, Thornwood, NY, USA) with a C-Apo $\times 40$ water-immersion objective. An excitation wave length of $488 \mathrm{~nm}$ with an LP $505 \mathrm{~nm}$ emission filter was used to detect GFP and FITC, and a wavelength of $568 \mathrm{~nm}$ with an LP $590 \mathrm{~nm}$ emission filter was used to detect Alexa Fluor 594. Images were analyzed with the LSM-510 Image Browser Software.

\section{Fluorescence-activated cell sorting analysis}

FADD-deficient cells transfected with the pEGF-C2 empty vector, WT-GFP FADD, NLS Mutant-FADD or NES MutantFADD-transfected cells were treated $18 \mathrm{~h}$ post-transfection with or without $500 \mathrm{ng} / \mathrm{ml}$ of anti-human Fas IgM CH-11 for $1 \mathrm{~h}$. Flow-cytometric analyses for changes in cell volume were examined on a Becton Dickinson FACSort using CellQuest (BD Biosciences) software through forward-scatter light analyses of cells, as reported previously. ${ }^{32}$ For examination of the GFP-positive cell populations, wave lengths of 488 and $530 \mathrm{~nm}$ were used for excitation and emission, respectively. To ensure that the amount of cell death was quantified on the positively transfected cell population exclusively, we determined analyse by gating on the GFP-positive cell population. Results were analyzed with Student's $t$-test for statistical significance.

\section{References}

1. Locksley RM, Killeen $\mathrm{N}$ and Lenardo MJ (2001) The TNF and TNF receptor superfamilies: integrating mammalian biology. Cell 104: 487-501

2. Chinnaiyan AM, O'Rourke K, Tewari M and Dixit VM (1995) FADD, a novel death domain-containing protein, interacts with the death domain of Fas and initiates apoptosis. Cell 81(4): 505-512

3. Strasser A and Newton K (1999) FADD/MORT1, a signal transducer that can promote cell death or cell growth. Int. J. Biochem. Cell Biol. 31: 533-537

4. Hsu H, Shu HB, Pan MG and Goeddel DV (1996) TRADD-TRAF2 and TRADD-FADD interactions define two distinct TNF receptor 1 signal transduction pathways. Cell. 84: 299-308

5. Schneider P, Thome M, Burns K, Bodmer JL, Hofmann K, Kataoka T, Holler N and Tschopp J (1997) TRAIL receptors 1 (DR4) and 2 (DR5) signal FADDdependent apoptosis and activate NF-kappaB. Immunity 7(6): 831-836

6. Boldin MP, Varfolomeev EE, Pancer Z, Mett IL, Camonis JH and Wallach D (1995) A novel protein that interacts with the death domain of Fas/APO1 contains a sequence motif related to the death domain. J. Biol. Chem. 270: 7795-7798

7. Kischkel FC, Hellbardt S, Behrmann I, Germer M, Pawlita M, Krammer PH and Peter ME (1995) Cytotoxicity-dependent APO-1 (Fas/CD95)-associated proteins form a death-inducing signaling complex (DISC) with the receptor. EMBO J. 14(22): 5579-5588

8. Alnemri ES, Livingston DJ, Nicholson DW, Salvesen G, Thornberry NA, Wong WW and Yuan J (1996) Human ICE/CED-3 protease nomenclature. Cell 87(4): 171

9. Chinnaiyan AM, Tepper CG, Seldin MF, O'Rourke K, Kischkel FC, Hellbardt S, Krammer PH, Peter ME and Dixit VM (1996) FADD/MORT1 is a common mediator of CD95 (Fas/APO-1) and tumor necrosis factor receptor-induced apoptosis. J. Biol. Chem. 271(9): 4961-4965

10. Zhang J, Cado D, Chen A, Kabra NH and Winoto A (1998) Fas-mediated apoptosis and activation-induced T-cell proliferation are defective in mice lacking FADD/Mort1. Nature 392: 296-300

11. Hueber AO, Zornig M, Bernard AM, Chautan M and Evan G (2000) A dominant negative Fas-associated death domain protein mutant inhibits proliferation and leads to impaired calcium mobilization in both T-cells and fibroblasts. J. Biol. Chem. 275: 10453-10462 
12. Newton K, Harris AW, Bath ML, Smith KG and Strasser A (1998) A dominant interfering mutant of $F A D D / M O R T 1$ enhances deletion of autoreactive thymocytes and inhibits proliferation of mature T lymphocytes. EMBO J. 17: $706-718$

13. Newton K, Harris AW and Strasser A (2000) FADD/MORT1 regulates the preTCR checkpoint and can function as a tumour suppressor. EMBO J. 19: 931-941

14. Walsh CM, Wen BG, Chinnaiyan AM, O'Rourke K, Dixit VM and Hedrick SM (1998) A role for FADD in T cell activation and development. Immunity 8: 439-449

15. Scaffidi C, Volkland J, Blomberg I, Hoffmann I, Krammer PH and Peter ME (2000) Phosphorylation of FADD/ MORT1 at serine 194 and association with a 70- kDa cell cycle-regulated protein kinase. J. Immunol. 164: 1236-1242

16. Bodmer JL, Holler N, Reynard S, Vinciguerra P, Schneider P, Juo P, Blenis J and Tschopp J (2000) TRAIL receptor-2 signals apoptosis through FADD and caspase-8. Nat. Cell Biol. 2(4): 241-243

17. Gomez-Angelats M and Cidlowski JA (2001) PKC inhibits FADD recruitment and DISC formation during FAS/CD95 induced apoptosis. J. Biol. Chem. 276 44944-44952

18. Scaffidi C, Kischkel FC, Krammer PH and Peter ME (2000) Analysis of the CD95 (APO-1/Fas) death-inducing signaling complex by high-resolution twodimensional gel electrophoresis. Methods Enzymol. 322: 363-373

19. Wieder T, Essmann F, Prokop A, Schmelz K, Schulze-Osthoff K, Beyaert R, Dorken B and Daniel PT (2001) Activation of caspase-8 in drug-induced apoptosis of B-lymphoid cells is independent of CD95/Fas receptor-ligand interaction and occurs downstream of caspase-3. Blood 97(5): 1378-1387

20. Qutob MS, Bhattacharjee RN, Pollari E, Yee SP and Torchia J (2002) Microtubule-dependent subcellular redistribution of the transcriptional coactivator p/CIP. Mol. Cell Biol. 22: 6611-6626

21. Zhang J and Winoto A (1996) A mouse Fas-associated protein with homology to the human Mort1/FADD protein is essential for Fas-induced apoptosis. Mol. Cell Biol. 16: 2756-2763
22. Jans DA, Xiao CY and Lam MH (2000) Nuclear targeting signal recognition: a key control point in nuclear transport?. Bioessays 22: 532-544

23. Siegel RM, Martin DA, Zheng L, Ng SY, Bertin J, Cohen J, Lenardo MJ (1998) Death-effector filaments: novel cytoplasmic structures that recruit caspases and trigger apoptosis. J. Cell Biol. 141(5): 1243-1253

24. Nakielny S and Dreyfuss G (1999) Transport of proteins and RNAs in and out of the nucleus. Cell 99: 677-690

25. Fischer U, Huber J, Boelens WC, Mattaj IW and Luhrmann R (1995) The HIV- 1 Rev activation domain is a nuclear export signal that accesses an export pathway used by specific cellular RNAs. Cell 82: $475-483$

26. Matsumura H, Shimizu $Y$, Ohsawa $Y$, Kawahara A, Uchiyama $Y$ and Nagata $S$ (2000) Necrotic death pathway in Fas receptor signaling. J. Cell Biol. 151: $1247-1256$

27. Newton K, Kurts C, Harris AW and Strasser A (2001) Effects of a dominant interfering mutant of FADD on signal transduction in activated T cells. Curr Biol. 11: $273-276$

28. Juo P, Woo MS, Kuo CJ, Signorelli P, Biemann HP, Hannun YA and Blenis J (1999) FADD is required for multiple signaling events downstream of the receptor Fas. Cell Growth Differ. 10(12): 797-804

29. Wyllie AH (1980) Glucocorticoid-induced thymocyte apoptosis is associated with endogenous endonuclease activation. Nature 284: 555-556

30. Morgan M, Thorburn J, Pandolfi PP and Thorburn A (2002) Nuclear and cytoplasmic shuttling of TRADD induces apoptosis via different mechanisms. J. Cell Biol. 157: 975-984

31. Burnstein KL, Jewell CM, Sar M and Cidlowski JA (1994) Intragenic sequences of the human glucocorticoid receptor complementary DNA mediate hormoneinducible receptor messenger RNA down-regulation through multiple mechanisms. Mol. Endocrinol. 8: 1764-1773

32. Gomez-Angelats M, Bortner CD and Cidlowski JA (2000) PKC inhibits Fasinduced apoptosis through modulation of the loss of potassium and cell shrinkage. A role for PKC upstream of caspases. J. Biol. Chem. 275: 19609-19619 